Dr. med.

Brigitte Moreano Stellvertretende Chefredakteurin brigitte.moreano@ springer.com

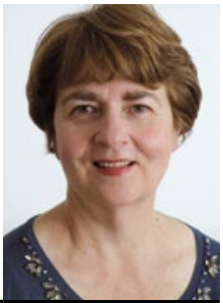

Verwandte mit Darmkrebs?

Häufig kognitive Defizite

\section{9/11-Ersthelfer: Droht nach der PTBS die Demenz?}

Eine posttraumatische Belastungsstörung (PTBS) erhöht möglicherweise das Risiko einer kognitiven Beeinträchtigung. Darauf weisen Untersuchungen hin, die nach den New Yorker Terroranschlägen bei Laienhelfern gemacht wurden. Jeder Fünfte von ihnen hatte eine PTBS entwickelt. Einer Stichprobenuntersuchung zufolge war jeder Siebte aus dieser Gruppe von kognitiven
Störungen betroffen: Ein 2014/2015 bei 818 Helfern mit PTBS durchgeführter Test hatte bei 104 Patienten $(12,8 \%)$ eine kognitive Beeinträchtigung und bei 10 (1,2\%) eine mögliche Demenz gezeigt. „Das sind schwindelerregende Zahlen in Anbetracht dessen, dass das Durchschnittsalter der Helfer bei 53 Jahren liegt“, so die Autoren.

- Alzheimer's \& Dementia 2016; 4:67-75

\title{
Acne tarda
}

\section{Lässt Stress späte Pickel sprießen?}

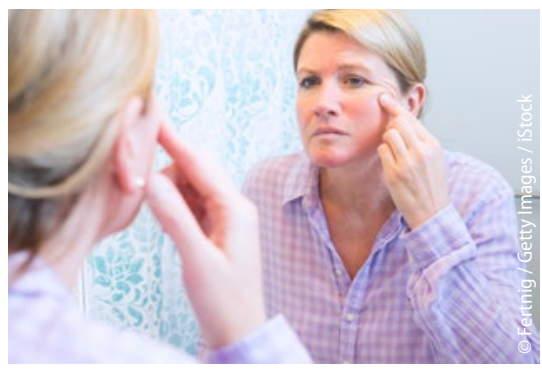

Wenn Frauen im Alter über 25 (noch) an Akne leiden, kann das mit ihrer Lebensführung zusammenhängen. 248 Patientinnen (mittleres Alter 32 Jahre) mit Late-onset-Ak- ne wurden mit 270 Frauen ohne Akne verglichen. Neben erwartbaren Risikofaktoren wie Akne in der Adoleszenz oder Hirsutismus fanden sich auch unerwartete Zusammenhänge: Die Aknepatientinnen waren öfter als die Kontrollgruppe als Büroangestellte tätig $(\mathrm{OR}=2,24)$ und empfanden ihren psychischen Stress häufiger als hoch oder sehr hoch $(\mathrm{OR}=2,95)$. Zudem ernährten sie sich öfter ungesund mit wenig Obst, Gemüse und Fisch. Ein Zusammenhang mit dem Konsum von Milch fand sich im Gegensatz zur Pubertätsakne nicht.

- JAAD 2016; online 16. August 2016

\section{Rezidivierende Clostridium-difficile-Infektionen Darmflora reloaded}

Durch eine kolonoskopische Transplantation frischen Stuhls von einem gesunden Spender konnten in einer US-Studie weitere C.-difficile-Infektionen bei 93,5\% der Patienten mit mehrfachen Rezidiven verhindert werden. 22 Patienten erhielten den frischen Stuhl eines gesunden Spenders und 24 Probanden ihren eigenen Stuhl (Kontrollgruppe). 90,9\% der Patienten, die

eine Stuhltransplantation von einem Fremdspender erhalten hatten, wurden nach acht Wochen als geheilt eingestuft. In der Kontrollgruppe lag diese Quote bei $62,5 \%$. Alle neun Patienten, die nach autologer Stuhltransplantation ein Rezidiv erlebten, wechselten in die Spendergruppe und galten danach ebenfalls als geheilt.

- Ann Intern Med 2016, online 23. August; doi: 10.7326/M16-0271
Dann lohnt sich die Koloskopie besonders

Wird bei familiär erhöhtem Darmkrebsrisiko regelmäßig koloskopiert, ist das Erkrankungsrisiko sogar geringer als in der Allgemeinbevölkerung ohne Screening. Darauf deutet eine Fall-Kontroll-Studie des DKFZ Heidelberg hin. Danach ist das Darmkrebsrisiko um etwa 70\% erhöht, wenn ein Angehöriger ersten Grades betroffen ist, bei regelmäßigen Screening liegt das Risiko jedoch nur halb so hoch wie in der nicht darmgespiegelten Allgemeinbevölkerung. In der Studie waren Angaben von mehr als 4.300 Darmkrebspatienten und 3.100 Kontrollpersonen aus der Allgemeinbevölkerung analysiert worden.

- Int J Cancer 2016, online 26. Juli; doi: 10.1002/ijc.30284

\section{Schlechte Stoffwechselprofile}

\section{Typ-1-Diabetiker zum Rauchstopp motivieren}

Raucher haben signifikant schlechtere Blutzuckereinstellungen als Nichtraucher $\left(\mathrm{HbA}_{1 \mathrm{c}} 8,5 \%\right.$ vs. 7,9\%). Dennoch gelingt es vielen Typ1-Diabetikern nicht, mit dem Rauchen aufhören. Daten aus der deutsch-österreichischen Diabetes-Patienten-Verlaufsdokumentation (9.862 Patienten) zeigen, dass $24,3 \%$ der Typ-1-Diabetiker über 18 Jahre mindestens eine Zigarette am Tag rauchen. In den USA dagegen sind laut T1D Exchange Registry (10.579 Patienten) nur 10\% der Typ-1-Diabetiker Raucher. 18,1\% der US-Diabetiker gaben an, sie hätten mit dem Rauchen aufgehört, während dies hierzulande nur 5,1\% geschafft hatten. - Diab Care 2016, online 16. August; doi: 10.2337/dc16-0845/-/DC1 